\title{
A GLOBAL ANALYSIS OF THE GENERALIZED SITNIKOV PROBLEM
}

\author{
STEVEN R. CHESLEY \\ Dipartimento di Matematica, Universitá di Pisa, 56127 Pisa, Italy; \\ E-mail: chesley@dm.unipi.it
}

\begin{abstract}
The isosceles three-body problem with Sitnikov-type symmetry has been reduced to a two-dimensional area-preserving Poincare map depending on two parameters: the mass ratio, and the total angular momentum. The entire parameter space is explored, contrasting new results with ones obtained previously in the planar (zero angular momentum) case. The region of allowable motion is divided into subregions according to a symbolic dynamics representation. This enables a geometric description of the system based on the intersection of the images of the subregions with the preimages. The paper also describes the regions of allowable motion and bounded motion, and discusses the stability of the dominant periodic orbit.
\end{abstract}

\section{Introduction}

The isosceles three-body problem has been studied for a variety of reasons and for more than one hundred years. Early researches concerned the possible isosceles configurations (Fransén, 1895), the necessary conditions for the isosceles symmetry to be preserved (Wilczynski, 1913), and the integrability of certain cases (Macmillan, 1913). Later Sitnikov (1961) used a special case of this problem to prove the existence of so-called oscillatory motion - unbounded oscillations with no escape. In recent decades considerable attention has focused on the triple collision manifold of the planar isosceles problem (e.g., Simó and Martinez, 1988).

In the present work the isosceles problem is used as an example to demonstrate a method of global analysis based on a Poincaré map. In this method one uses the mapping to investigate the evolution of entire areas under a single iteration, rather than examining the behavior of a few points on the surface of section under hundreds or thousands of iterations as is typically done. This new approach permits insight into the structure and evolution of the chaotic regions (seas) of the phase space. On the other hand the traditional approach shows very clearly the structure and extent of the stable regions (islands). In this sense the new method is very much complementary to the traditional approach.

The isosceles problem is obtained by applying certain special symmetries to the initial conditions. The present formulation permits the representation of both the Sitnikov and planar configurations, which are usually modelled separately. In this formulation there are two free parameters which govern the dynamics, a mass ratio $\alpha$ and the angular momentum of the binary $c$. The Sitnikov configuration consists of a symmetric binary system with nonzero angular momentum and a secondary mass moving for all time along the unique line that is perpendicular to the plane of the binary and passes through the center of mass of the binary. In this configuration, the three masses always form an isosceles triangle, and the triangle is always rotating about its axis of symmetry. (See Fig. 1.) If the angular momentum of the binary is zero then the triangle is confined to an invariable plane. This is the planar isosceles configuration. 
These two configurations are most importantly distinguished by the presence or absence of collisions. In the planar case the binary motion is rectilinear since $c=0$, thus there is a binary collision at every pericenter. If the binary collision is coincident with a crossing of the syzygy line by the third mass then we have a triple collision. In contrast, the Sitnikov case has no possibility of binary collision, hence triple collision is clearly also excluded.

In a previous paper (hereafter Paper I) Zare and Chesley (1998) studied carefully the case of planar motion with three equal masses. In that paper the tools and principles used in the present study are described in much greater detail than is possible here. Later (Paper II) Chesley and Zare (1998) expanded the study to include all possible mass ratios, while maintaining the planar condition $(c=0)$. Finally, in this paper, the entire parameter space is explored, considering all possible values of angular momentum and mass ratio. New results are put into contrast with properties obtained in the previous papers. This paper also presents some more general results on the problem, including a discussion of the allowable motions, and a global analysis of the stability properties of the dominant periodic orbit.

\section{The General Isosceles Problem}

A full development of the system equations can be found in (Chesley, 1998), but for brevity let us begin with the Hamiltonian in physical variables

$$
\mathcal{H}=\frac{1}{2}\left(p_{1}^{2}+\frac{p_{2}^{2}}{\alpha}\right)-\frac{G m}{4 q_{1}}-\frac{G m_{3}}{\sqrt{q_{1}^{2}+q_{2}^{2}}}+\frac{c^{2}}{2 q_{1}^{2}} .
$$

Here $\left(q_{1}, q_{2}\right)$ are the distances of a binary element and the secondary, respectively, from the binary mass center (see Fig. 1 ), and $\left(p_{1}, p_{2}\right)$ are the corresponding conjugate momenta. The constant of gravitation is given by $G$, while $m$ is the mass of each of the binary components and $m_{3}$ is the mass of the secondary or "third mass". We also have the binary angular momentum $c$, and the ratio of the secondary mass to the total mass $\alpha=m_{3} /\left(2 m+m_{3}\right), 0<\alpha \leq 1$. We will use the value of the Hamiltonian to represent the system energy $h=\mathcal{H}$.

Remark: The Hamiltonian formulation does not permit the analysis of the classical Sitnikov problem, which is the restricted case $(\alpha=0)$. This fact can be clearly seen from Eq. (1).

For reasons which will become apparent later, we shall apply the following normalization of units throughout

$$
G=1, \quad m+4 m_{3}=1 / 2, \quad h=-1 / 8 .
$$

There is no loss of generality with this normalization. The system fundamentally has the following parameters: the constant of gravity $G$, the energy $h$, the angular momentum $c$, and two mass parameters $m$ and $m_{3}$. Selecting the units of mass, 


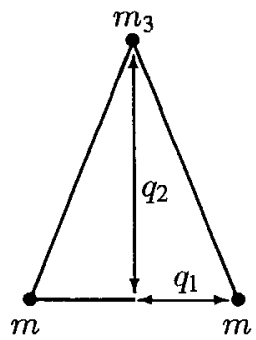

Fig. 1. Geometry of the isosceles problem. Note that the binary rotates about the axis of symmetry if $c>0$.

length, and time according to the normalization given above fixes $G, h$, and one of the mass parameters. This leaves the angular momentum $c$, and the other mass parameter, which we may choose to be $\alpha$, as the only free parameters. Selection of $\alpha$ within this normalization has the effect of determining the mass values according to the identities $m=(1-\alpha) /(1+7 \alpha) / 2$ and $m_{3}=\alpha /(1+7 \alpha)$.

Note: This Hamiltonian has been scaled from the usual three-body energy expression by a factor $2 m$ to allow a simpler presentation. This means that the usual system energy and usual binary angular momentum are given by $2 m h$ and $2 m c$, respectively.

\subsection{REgULARIZATION}

If $c=0$ the equations of motion stemming from Eq. (1) are singular at the binary collision $\left(q_{1}=0, q_{2} \neq 0\right)$ and at the triple collision $\left(q_{1}=q_{2}=0\right)$. (If $c$ is small but nonzero the equations are near-singular at binary pericenter.) The singularity at or near the binary collision may be removed by the classical LeviCivita regularization. In order to preserve the Hamiltonian form, we shall adapt the regularization to the extended phase space. First, we introduce the generating function $W=p_{1} Q_{1}^{2}+p_{2} Q_{2}$, and the associated canonical transformation

$$
q_{i}=\frac{\partial W}{\partial p_{i}}, \quad P_{i}=\frac{\partial W}{\partial Q_{i}}, \quad i=1,2,
$$

leading to the Hamiltonian

$$
\mathcal{H}=\frac{1}{2}\left(\frac{P_{1}^{2}}{4 Q_{1}^{2}}+\frac{P_{2}^{2}}{\alpha}\right)-\frac{G m}{4 Q_{1}^{2}}-\frac{G m_{3}}{\sqrt{Q_{1}^{4}+Q_{2}^{2}}}+\frac{c^{2}}{2 Q_{1}^{4}} .
$$

Under these new variables one may construe $\left(Q_{1}, P_{1}\right)$ as the state vector of the binary, and $\left(Q_{2}, P_{2}\right)$ as the state vector of the third mass.

Introduction of the auxiliary variables $Q_{0}=t, P_{0}=-h$, and $d \tau=d t / Q_{1}^{2}$ provides the new equations of motion

$$
\frac{d Q_{i}}{d \tau}=\frac{\partial \Gamma}{\partial P_{i}}, \quad \frac{d P_{i}}{d \tau}=-\frac{\partial \Gamma}{\partial Q_{i}}, \quad i=0,1,2
$$


where the new Hamiltonian function in the extended phase space $\Gamma=Q_{1}^{2}\left(P_{0}+\mathcal{H}\right)$ is given by

$$
\Gamma=\frac{1}{2}\left(\frac{P_{1}^{2}}{4}+\frac{P_{2}^{2} Q_{1}^{2}}{\alpha}\right)-\frac{G m}{4}-\frac{G m_{3} Q_{1}^{2}}{\sqrt{Q_{1}^{4}+Q_{2}^{2}}}+P_{0} Q_{1}^{2}+\frac{c^{2}}{2 Q_{1}^{2}} .
$$

On every trajectory we have the energy integral $\Gamma \equiv 0$, and the equations of motion are now regular at the binary collision $\left(Q_{1}=0, Q_{2} \neq 0\right)$.

\subsection{REgION OF POSSIBLE MOTION IN THE CONFIGURATION SPACE}

A general theory has been developed by Zare (1976) to obtain the regions of possible motion in the configuration space, and in particular the bifurcation sets of their topological classification (Zare, 1977). For the present problem we will follow the theory to obtain the maximum possible angular momentum for a given energy and to identify regions of bounded motion in the parameter space.

Starting with Eq. (1) we obtain the inequality

$$
h \geq-\frac{G m}{4 q_{1}}-\frac{G m_{3}}{q_{1} \sqrt{1+q_{2}^{2} / q_{1}^{2}}}+\frac{c^{2}}{2 q_{1}^{2}} .
$$

By introducing the new variable

$$
\theta=\arctan \frac{q_{2}}{q_{1}}, \quad-\frac{\pi}{2} \leq \theta \leq \frac{\pi}{2},
$$

this inequality can be recast as

$$
\psi\left(q_{1}, \theta\right)=4|h| q_{1}^{2}-G\left(m+4 m_{3} \cos \theta\right) q_{1}+2 c^{2} \leq 0,
$$

where we have assumed $h<0$. The variables here represent the configuration $(\theta)$ and the scale $\left(q_{1}\right)$ of the triangle formed by the three bodies. Note that $\theta=0$ implies syzygy, and $\theta= \pm \pi / 2$ implies either binary collision or the escape of the third mass. From Eq. (4) it is immediate that if $c \neq 0$ then $q_{1}>0$ for all time, hence the binary collision is forbidden. The possible range for $q_{1}$ is obtained from the quadratic equation where the discriminant is given by

$$
\Delta(\theta)=G^{2}\left(m+4 m_{3} \cos \theta\right)^{2}-32 c^{2}|h| \geq 0 .
$$

Equation (5) provides the totality of possible configurations, independent of scale. Notice that $c^{2}|h|$, sometimes referred to as the Zare integral, appears as an essential parameter. It is easy to show that $\Delta(\theta)$ has a maximum at $\theta=0$ with maximal value

$$
\Delta(0)=G^{2}\left(m+4 m_{3}\right)^{2}-32 c^{2}|h| \geq 0 .
$$

From this we obtain the critical value

$$
c^{2}|h| \leq\left(c^{2}|h|\right)_{1}=G^{2}\left(m+4 m_{3}\right)^{2} / 32 .
$$


If we apply the normalization of units given by Eq. (2) then we obtain the permissible range of angular momentum: $0 \leq c \leq \frac{1}{4}$. (We shall assume $c \geq 0$, the direction of rotation being irrelevant to the dynamics.)

Remark: The maximum value of $c$ reflects an important orbit. Note that this value requires $p_{1}=p_{2} \equiv 0$ and $\theta \equiv 0$, which implies $q_{2} \equiv 0$. In this case we have $q_{1} \equiv \frac{1}{2}$ under our normalization according to Eq. (4). So for $c=\frac{1}{4}$ the motion is limited to a single point in the phase space. This orbit is the so-called Euler solution. Here the binaries rotate on circular orbits with radius $q_{1}=\frac{1}{2}$ and the third mass remains forever fixed at the binary center of mass.

\subsubsection{The Region of Bounded Motion}

If the angular momentum is large enough the third mass cannot escape to infinity, while the binary remains bounded according to Eq. (4). This implies bounded motion for all particles for all time. To determine the smallest value of angular momentum where bounded motion is assured, let us continue by recasting Eq. (5) as

$$
\cos \theta \geq \frac{\sqrt{32 c^{2}|h|}-G m}{4 G m_{3}} .
$$

From this we can see that for $c^{2}|h|>\left(c^{2}|h|\right)_{1}$ there exist no possible configurations, and for $c^{2}|h|=\left(c^{2}|h|\right)_{1}$ only $\theta=0$ is possible. But for $c^{2}|h|<\left(c^{2}|h|\right)_{1}$ the possible configurations are $|\theta| \leq \theta_{\max }$, where $\theta_{\max }$ is obtained from Eq. (6). It is clear that $\theta_{\max }=\pi / 2$ at the critical value

$$
\left(c^{2} h\right)_{2}=G^{2} m^{2} / 32 \text {. }
$$

Recall that $\theta= \pm \pi / 2$ corresponds to escape of $m_{3}$ if $c>0$. Therefore the third mass may go to infinity for $c^{2}|h| \leq\left(c^{2}|h|\right)_{2}$ since the possible configurations are $-\pi / 2 \leq \theta \leq \pi / 2$. But for $c^{2}|h|>\left(c^{2}|h|\right)_{2}$ we have $|\theta|<\pi / 2$, and escape is impossible. After normalization of units according to Eq. (2) we have the final result

$$
c \geq \frac{1-\alpha}{4(1+7 \alpha)} \Longrightarrow \text { bounded motion. }
$$

The corresponding region of the parameter space is plotted in Fig. 2.

\subsection{THE Boundaries of THE PARAMETER SPACE}

Each of the boundaries for the space of parameters $(\alpha, c) \in(0,1] \times\left[0, \frac{1}{4}\right]$ has a special significance. (See Fig. 2.) 


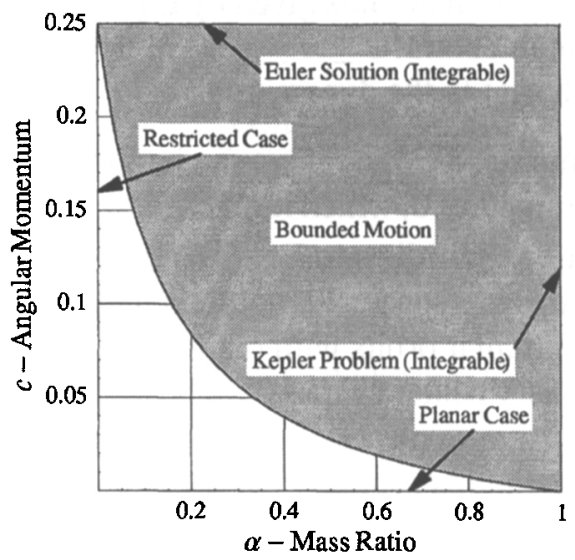

Fig. 2. Region of bounded motion in the $(\alpha, c)$-space of parameters. The special significance of each boundary is also depicted.

1. $c=0$. This is the planar isosceles problem. This boundary was the focus of Paper II, and the point on this boundary at $(\alpha, c)=\left(\frac{1}{3}, 0\right)$ was studied in detail in Paper I.

2. $c=\frac{1}{4}$. The motion here corresponds to an integrable solution of the general three-body problem-the Euler solution discussed above. The motion is reduced to a single fixed point in the phase space.

3. $\alpha=0$. Here the third mass is zero-the classical (restricted) Sitnikov problem. This is the only boundary that cannot be treated by the present formulation of the equations of motion. As one approaches this boundary, hyperbolic escape of $m_{3}$ approaches full measure. (See Paper II).

4. $\alpha=1$. On this boundary all of the mass is concentrated in the third body. This leads to Keplerian motion, so here again the solution is integrable. This is the double Kepler problem described in Paper II. Here every point is a fixed point under the Poincare mapping described in the next section.

\section{Reduction to a Poincaré Mapping}

The properties of the Poincare map in the planar isosceles problem have been discussed extensively in Papers I and II. The primary aim of the present paper is to describe certain new properties which have appeared after extending the investigation to include nonzero angular momentum. For this reason, and for reasons of space, only a cursory introduction to the methods employed previously is presented here. For a comprehensive discussion, the interested reader is referred to Paper I, Paper II, and (Chesley, 1998).

To arrive at the desired surface of section we eliminate $P_{2}$ using the integral of energy, and take as a section the plane $Q_{2}=0$ (syzygy crossing). Thus one obtains a mapping in the $\left(Q_{1}, P_{1}\right)$-plane. This is an excellent surface of section 


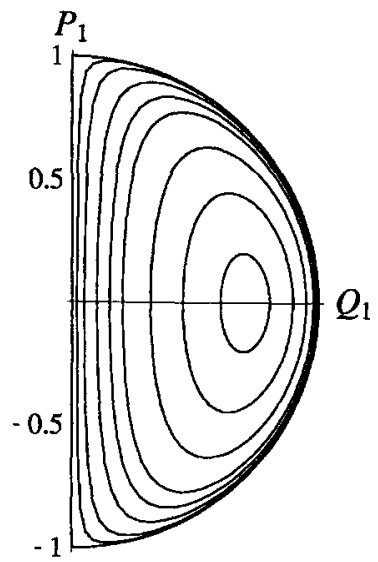

Fig. 3. Boundary curves for the regions of possible motion $A$ in the Poincare section for varying angular momentum. The depicted curves correspond to the following values of $c$ : $\{0,0.01,0.025,0.05,0.075,0.10,0.15,0.20,0.24\}$.

because almost every isosceles trajectory intersects it, with the only exceptions being certain isolated triple collision orbits. The region of allowable motion $A$ depends only upon the angular momentum $c$ according to

$$
A=\left\{\left(Q_{1}, P_{1}\right) \mid Q_{1}^{2}+P_{1}^{2}+\frac{4 c^{2}}{Q_{1}^{2}}<1, Q_{1}>0\right\} .
$$

We specify $Q_{1}>0$ without loss of generality since $q_{1}=Q_{1}^{2} \geq 0$, and on this section $Q_{1}=0$ implies a triple collision condition. Every point in $A$ represents a unique initial condition with $Q_{2}=0$ and $P_{2} \geq 0$ computed from the energy integral. The bounding curves for varying levels of $c$ are depicted in Fig. 3 .

For $c=0$ (the planar case) $A$ reaches its greatest extent forming a semicircular region of unit radius. (In fact, the somewhat curious normalization of units in Eq. (2) has been selected to this end.) In this case the straight boundary of $A$ (on which $Q_{1}=0$ ) corresponds to the triple collision state, while the semicircular boundary of $A$ corresponds to the collinear homothetic solution. For $c>0$ the boundary of $A$ corresponds to the Euler (collinear) solution.

The selection of this surface of section permits a useful physical interpretation of the mapping since $Q_{2}=0$ represents the syzygy configuration. Every trajectory started from a point in $A$ must return to another point in $A$ at the next syzygy crossing unless $m_{3}$ escapes or goes to triple collision. This may be viewed as a two-dimensional map $f: \bar{A} \rightarrow A$, where $\bar{A} \subset A$ is the set of points which neither escape nor lead to triple collision. Thus, for a given binary state $p_{0}=\left(Q_{1}, P_{1}\right)_{0} \in \bar{A}$, we define $f\left(p_{0}\right)$ to be the binary state $p_{1}=\left(Q_{1}, P_{1}\right)_{1} \in A$ taken at the next syzygy crossing. 

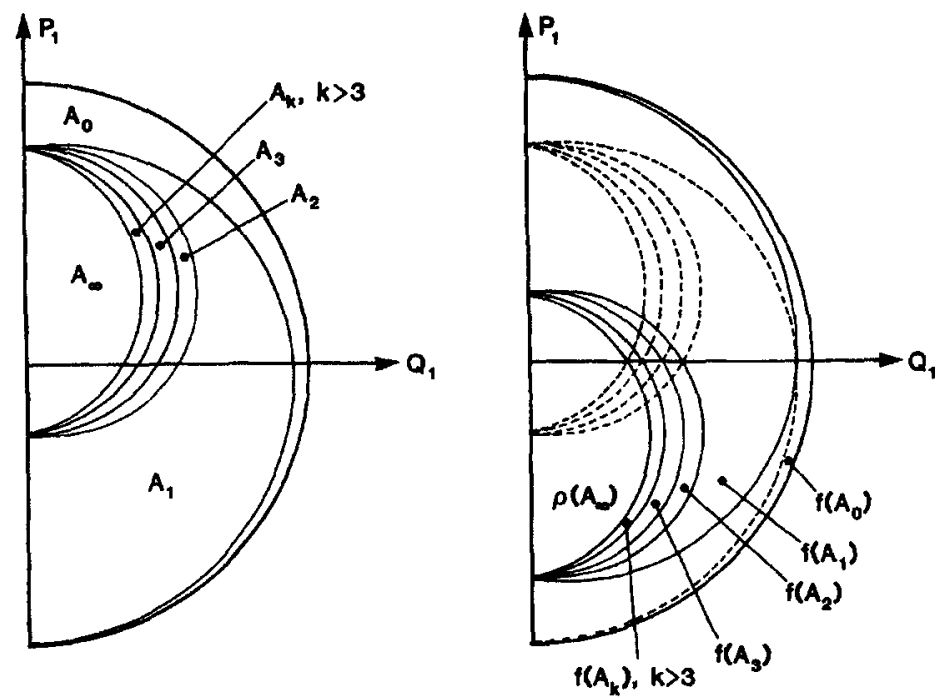

Fig. 4. The geometrical description of $f$ with $\alpha=\frac{1}{3}$ (equal masses) and $c=0$ (planar). The left diagram depicts the partitioning of $A$ into $A_{k},(k=0,1,2, \ldots)$. The right diagram depicts the images of the $A_{k}$. Here the preimages are shown with dashed lines to emphasize the intersections discussed in the text.

\subsection{PROPERTIES OF THE MAPPING}

The global description of $f$, which is given in greater detail in Paper I, proceeds as follows. Since each point in $\bar{A}$ must return for a subsequent syzygy crossing, we may assign to it a non-negative integer $k$ representing the number of binary pericenters which ensue before the next syzygy crossing. This will partition $A$ into open subsets $A_{k}$ where all points in each subset have the same assigned $k$ (see Fig. 4). Additionally, we denote the points which lead to hyperbolic escape of $m_{3}$ by $A_{\infty}$. Separating $A_{k}$ and $A_{k+1}$ is a locus of points $B_{k}$ which lead to a binary pericenter at the next syzygy (triple collision in the planar problem) after $k$ binary collisions. The boundary of $A_{\infty}$, which we denote by $B_{\infty}$, corresponds to a set of points leading to parabolic escape of $m_{3}$.

Due to the conservative nature of this system and the associated time reversibility we have a useful symmetry. Consider the reflection $\rho$ about the $Q_{1}$-axis in the $\left(Q_{1}, P_{1}\right)$-plane defined by

$$
\left(Q_{1}, P_{1}\right) \stackrel{\rho}{\longrightarrow}\left(Q_{1},-P_{1}\right) \text {. }
$$

Now $p$ can be considered as a reversal of the velocity, or, alternatively, a reversal of the arrow of time. Thus the backward orbit of the reflection of $p$ is the same as the reflection of the forward orbit of $p$ and it has the same number of binary collisions as the forward orbit of $p$. This leads to the identity $\rho f=f^{-1} \rho$. It also follows that for any $p \in A_{k}$, we have $\rho f(p) \in A_{k}$ or $f(p) \in \rho\left(A_{k}\right)$. That is

$$
f\left(A_{k}\right)=\rho\left(A_{k}\right) \text {. }
$$


Furthermore, if the orbit of $p$ corresponds to the escape of $m_{3}$ the orbit of $p(p)$ corresponds to the capture of $m_{3}$. If the orbit of $p$ terminates with a triple collision the orbit of $\rho(p)$ initiates with a triple ejection.

The identity (7) holds only for the $A_{k}$ and is not true for other sets in general. However, it provides a convenient means of obtaining the forward images $f\left(A_{k}\right)$. The asymmetry and the intersections of these subregions $A_{k}$ with the $Q_{1}$-axis are the necessary ingredients for the intersections $A_{j} \cap \rho\left(A_{k}\right)$ illustrated in Fig. 4. These intersections allow one to ascertain the existence and amount of communication between the $A_{k}$. They are also a necessary condition for the existence of period-one and -two orbits under $f$ as discussed in the next section.

This partitioning of $A$ into $A_{k}$ and $B_{k}$ is particularly conducive to a symbolic sequence representation of an orbit. We define the $n$th character in the symbolic sequence to be $k$ if $f^{n}(p) \in A_{k}$, for all integer $n$. Thus a sequence of $k$ 's corresponds to the sequence of $A_{k}$ 's visited under $f^{n}$ (in both directions of time). If $f^{n}(p)$ is not defined due to hyperbolic escape (capture) then we terminate the sequence on the right (left) with the symbol " $\infty$ ". If $f^{n}(p) \in B_{k}$ we may terminate the sequence with some appropriate symbol, say " $*_{k}$ ". These sequences and subsequences are very valuable in any effort to qualitatively categorize and characterize orbits.

\subsection{NEW RESULTS FOR $c>0$}

For $c=0$ the regions $A_{k}$ for $k \geq 2$ were crescent shaped (Fig. 4), but for $c>0$ they are segments of a spiral structure. Fig. 5 provides a particularly clear example of the spiral shape. In this figure there are two distinct curves along which the value of $k$ changes (the shading in the figure changes). The curve with an obvious spiral shape is the set of points for which the orbit returns to syzygy at pericenter. This spiral comprises the $B_{k}$, which were the orbits that returned to triple collision in the planar problem. The other, relatively straight, curve in Fig. 5 is the set of points for which the orbit begins at pericenter. In the planar case these were the points corresponding to triple collision. Geometrically what is happening is that, as the angular momentum increases above zero, the tips of the crescent-shaped $A_{k}$ of Fig. 4 bend inward to touch across this curve. On the other hand, as $c$ decreases to zero the curve diminishes to a segment on the $Q_{1}$-axis-the segment forming the border with $A_{\infty}$ in Fig. 4 .

As described above, for large enough values of $c$ there is no possibility of escape. However, being below that curve is only a necessary condition for escape; it is not sufficient. There must, however, be a curve-on or below the one providing the necessary condition-that gives the sufficient condition. This curve can be defined as the locus of parameter values for which the only escape orbits are parabolic. Above this curve $A_{\infty}$ has been destroyed. As the mass ratio or angular momentum continues to increase each $A_{k}$ is destroyed sequentially until only $A_{0}$ and $A_{1}$ survive. So for each $k \geq 2$ there is a curve in the parameter space marking the destruction of the corresponding $A_{k}$. This is quite intuitive because as $c$ increases, 


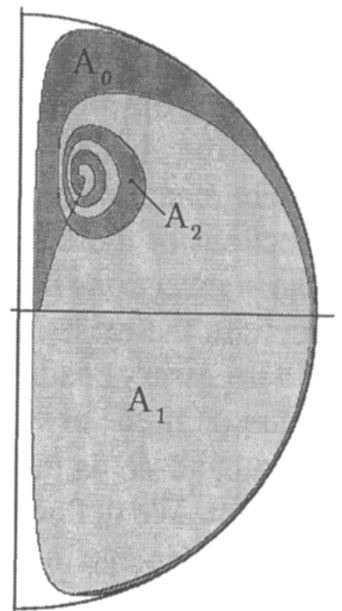

Fig. 5. Partitioning of $A_{k}$ at $\alpha=0.67, c=0.025$, showing the spiral effect described in the text. The axes are the same as Fig. 3.

the maximum ejections of the third body become more and more limited until $c=\frac{1}{4}$, at which point $Q_{2}(t) \equiv 0$. An approximate plot of the first and last curve is given in Fig. 6. The lower curve marks where $A_{\infty}$ is destroyed. The upper curve is where $A_{2}$-indeed all $A_{k}$, for $k \geq 2$-have been destroyed, and above this only $A_{0}$ and $A_{1}$ survive.

In terms of the symbolic dynamics, the destruction of $A_{\infty}$ is very important because it implies that all sequences are bi-infinite (except for a zero measure set of triple collision orbits). Furthermore, the symbolic alphabet becomes finite. These factors make the symbolic dynamics more tractable, and potentially more useful.

In Paper II we discussed a curious global bifurcation in the planar problem that occurred when, at a particular value of $\alpha$, all of the $A_{k}$ for $k \geq 2$ moved above the $Q_{1}$-axis. This "instantaneous" event, where an infinity of periodic and triple ejection-collision orbits are simultaneously destroyed, no longer occurs for $c>0$. The reason is that the $A_{k}$ are no longer crescent shaped, but rather they have the spiral shape discussed above. This means that the $A_{k}$ move above the $Q_{1}$-axis sequentially rather than simultaneously. Thus the intersections illustrated in Fig. 4 and the associated periodic orbits (as opposed to the regions themselves) are gradually destroyed as $c$ or $\alpha$ increase until all of the $A_{k}, k \geq 2$, are above the $Q_{1}$-axis as in the example of Fig. 5. The dashed curves in Fig. 6 reflect this movement, the global bifurcation occurring where they intersect at $c=0$.

\section{Stability of the Main Periodic Orbit}

In Paper I considerable attention was given to the main periodic orbit and the surrounding stable (quasi-periodic) region, where it was shown that this orbit has an important impact on the global dynamics. The orbit corresponds to the 


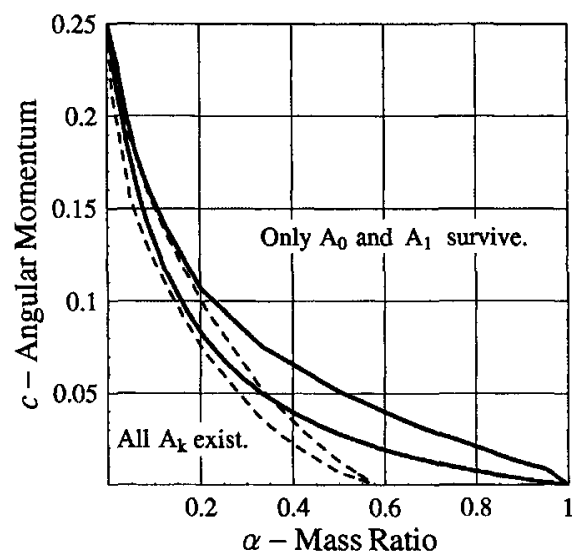

Fig. 6. Regions where particular $A_{k}$ survive. Below the lowest solid curve, all of the $A_{k}$ persist, including $A_{\infty}$. Above the upper solid curve, only $A_{0}$ and $A_{1}$ exist. The sequential elimination of the $A_{k}$ takes place between these two curves. The dashed curves depict the region where the $A_{k}$ move above the $Q_{1}$-axis, also sequentially.

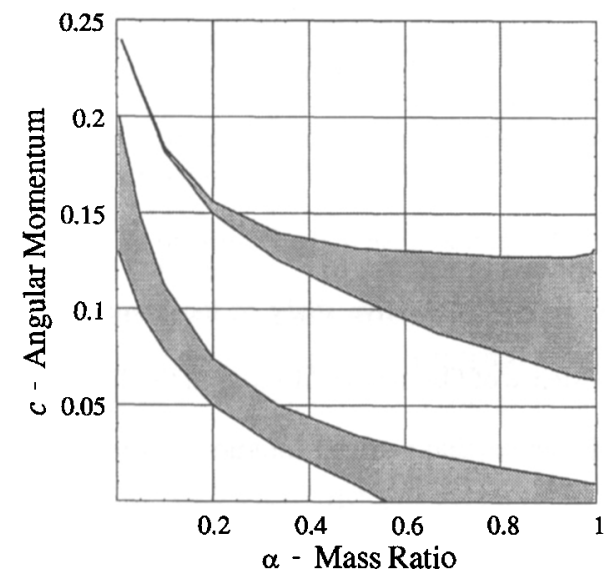

Fig. 7. Stability of the main periodic orbit. Unstable regions are shaded.

case when syzygy crossing occurs at binary apocenter, and there is exactly one binary pericenter between syzygy crossings. This means that triple interactions are minimized on this orbit, and the corresponding symbolic sequence for this orbit takes the form " $\ldots 1,1,1 \ldots$ ". In Paper II we showed that the main periodic orbit for the planar problem $(c=0)$ is stable for all values of $\alpha<0.563 \ldots$ Now, for completeness, let us briefly expand that discussion to the entire parameter space.

The author has identified through computer experiments two bands in $(\alpha, c)$ space where the main periodic orbit is unstable. (See Fig. 7.) Each of these bands is bounded above and below by a bifurcation curve. The uppermost bifurcation curve is of the ordinary period doubling type, while the three lowest curves represent 
inverse period doubling bifurcations. The path of the curves very close (within 0.005 ) to the limiting values of $\alpha$ is not known at present. An analytic treatment of these situations would likely yield definitive answers, but this must be left for future study. Recently, Dvorak and Sun (1997) have obtained similar results, although they have studied only the equal mass $\left(\alpha=\frac{1}{3}\right)$ case, and they have not computed orbits below approximately $c=0.05$ since their equations of motion are unregularized.

An investigation is underway on the stability of the main periodic orbit within the framework of the general three-body problem. The important question is whether there exist regions of quasi-periodic motion in the full phase space, implying at least the possibility of the existence of near-isosceles triple stellar systems.

\section{Acknowledgements}

The valuable contributions of Dr. K. Zare are gratefully acknowledged.

\section{References}

Chesley, S. and K. Zare: 1998, 'Bifurcations in the Mass Ratio of the Planar Isosceles Three-Body Problem'. In: A. E. Roy and B. A. Steves (eds.): Dynamics of Small Bodies in the Solar System.

Chesley, S. R.: 1998, 'The Isosceles Three-Body Problem: A Global Geometric Analysis'. Ph.D. thesis, University of Texas at Austin.

Dvorak, R. and Y. S. Sun: 1997, 'The Phase Space Structure of the Extended Sitnikov Problem'. Celest. Mech. Dyn. Astron. 67, 87-106.

Fransén, A. E.: 1895, 'Ett Specialfall Af Tre-Koppars-Problemet: Tva Himlakroppar Röra Sig Pa Lika Stora Afstand Fran Den Tredje'. Öfversigt af Kongl. Vetenskaps-Akademiens Förhandlingar 52, 783-805.

MacMillan, W. D.: 1913, 'An Integrable Case in the Restricted Problem of Three Bodies'. Astron. I. $27,11-13$.

Simó, C. and R. Martínez: 1988, 'Qualitative study of the planar isosceles three-body problem'. Celest. Mech. 41, 179-251.

Sitnikov, K.: 1961, 'The Existence of Oscillatory Motions in the Three-Body Problem'. Soviet Phys. Dokl. 5(4), 647-650.

Wilczynski, E. J.: 1913, 'Ricerche Geometriche Intorno al Problema Dei Tre Corpi'. Annali di Matematica, Ser. 3 21, 1-31.

Zare, K.: 1976, 'The Effect of Integrals on the Totality of Solutions of Dynamical Systems'. Celest. Mech. 14, 73-83.

Zare, K.: 1977, 'Bifurcation Points in the Planar Problem of Three Bodies'. Celest. Mech. 16, 35-38.

Zare, K. and S. Chesley: 1998, 'Order and Chaos in the Planar Isosceles Three-Body Problem'. Chaos $8(2), 475-494$. 